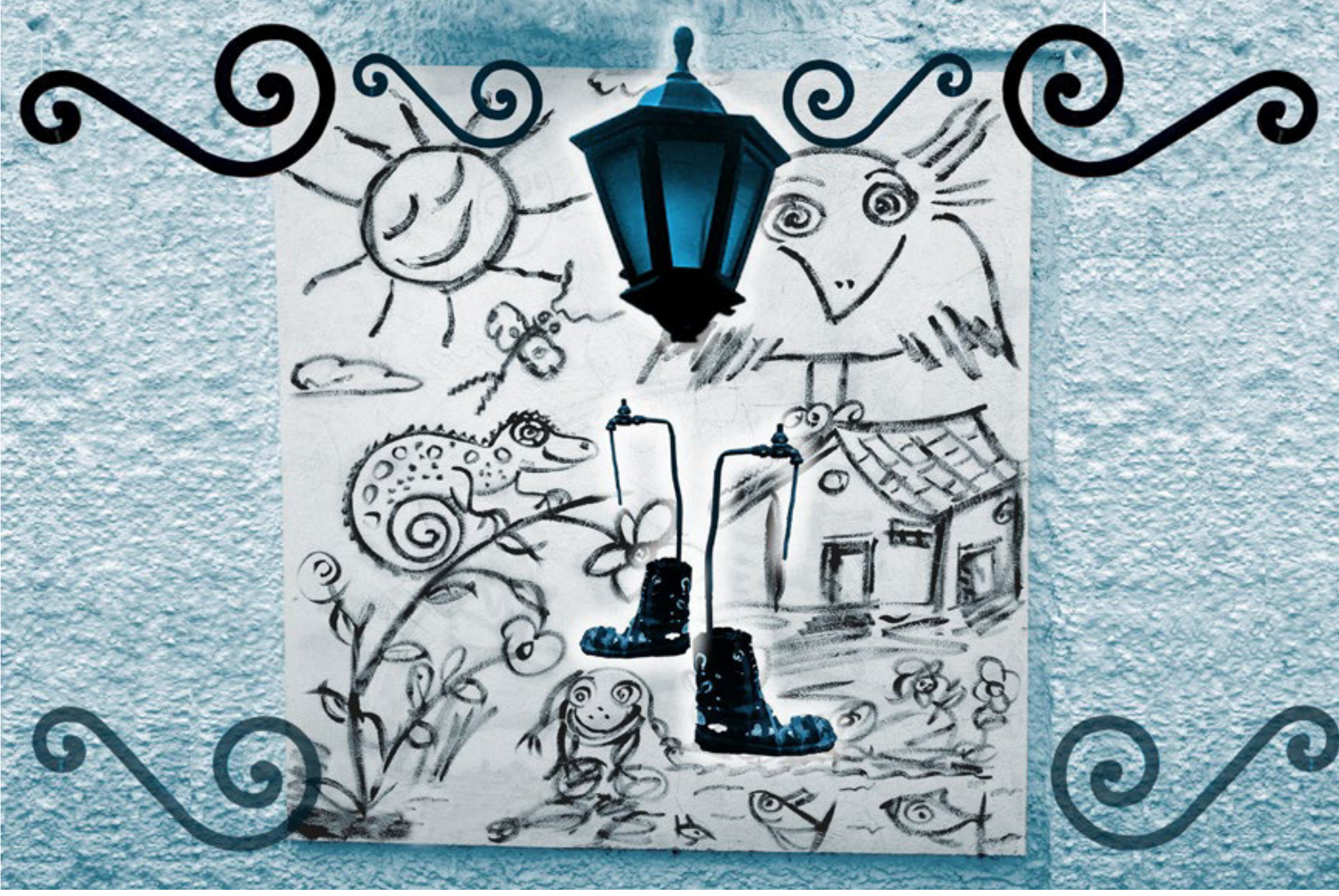

\title{
Validación del Índice del Derecho a la Educación (IDE) para Colombia, empleando la metodología Delphi
}

Validation of the Right to Education Index (IDE) for Colombia, using the Delphi Methodology Validação do Î́ndice do Direito à Educação (IDE) para a Colômbia, empleando a metodologia Delphi Hernando Bayona Rodríguez Arturo Harker Roa Camilo Ernesto López Guarín 


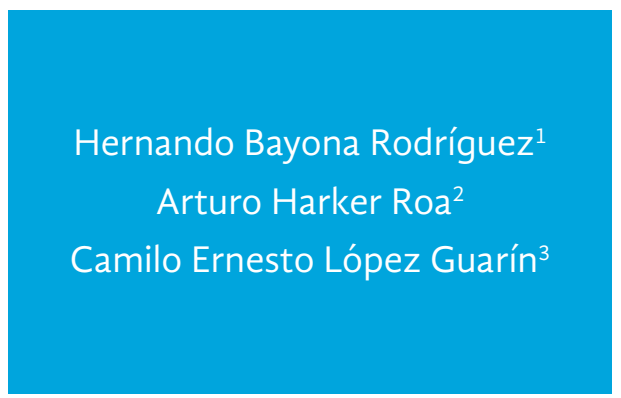

Doctor en economía, Universidad de los Andes. Profesor, Facultad de Educación, Universidad de Los Andes; Profesor Universidad Nacional de Colombia; correo electrónico: h.bayona28@ uniandes.edu.co

2. Doctor en Economía, Universidad de California. Profesor, Escuela de Gobierno Alberto Lleras Camargo, Universidad de Los Andes; correo electrónico: a.harker@uniandes.edu.co

3. Magíster en Ingeniería de Sistemas y Computación, Universidad Nacional de Colombia. Asistente de investigación, Facultad de Educación, Universidad de Los Andes; correo electrónico: ce.lopez@uniandes.edu.co

Fecha de recepción: 27 de noviembre de 2017 / Fecha de aprobación: 30 de marzo de 2018

\section{Resumen}

Elpresente documento expone los resultados de la validación del Índice del Derecho a la Educación (IDE) para Colombia. El IDE está compuesto por cuatro dimensiones: Disponibilidad, Accesibilidad, Adaptabilidad y Acepta-bilidad. La validación tiene como objetivo establecer qué indicadores dan cuenta de cada dimensión. Para llegar a un consenso se usó la metodología Delphi, implementada en un aplicativo Web. Este proceso contó con la participación de 158 expertos del sector educativo, quienes, en dos rondas, clasificaron cada uno de los 12 indicadores analizados en una de las cuatro dimensiones.

Palabras clave: Derecho a la educación, validación de indicadores, metodología Delphi, Índice del Derecho a la Educación, IDE.

\section{Abstract}

This document presents the results of the validation of the Right to Education Index (IDE) for Colombia. The IDE is composed of four dimensions: Availability, Accessibility, Adaptability and Acceptability. The validation aims to establish which indicators account for each dimension. To reach a consensus, the Delphi methodology was implemented in a Web application. This process was attended by 158 experts from the education sector, who, in two rounds, classified each of the 12 indicators analyzed in one of the four dimensions.

Keywords: Right to education, validation of indicators, delphi methodology, right to education index, IDE.

\begin{abstract}
Resumo
O presente documento expõe-se aos resultados da validação do Índice do Direito à Educação (IDE) para a Colômbia. El IDE está compilado por quatro dimensões: Disponibilidad, Accesibilidad, Adaptabilidad y Aceptabilidad. A validação tem por objetivo estabelecer indicadores de qualidade de cada dimensão. Para obter um consenso sobre a metodologia Delphi, implementada em um aplicativo Web. Este é um processo que conta com a participação de 158 especialistas do setor educativo, quienes, en dos rondas, clasificando cada um dos 12 indicadores analisados em uma das quatro dimensões.
\end{abstract}

Palavras-chave: Derecho à educação, validação de indicadores, metodologia Delphi, Índice da Educação à Educação, IDE. 


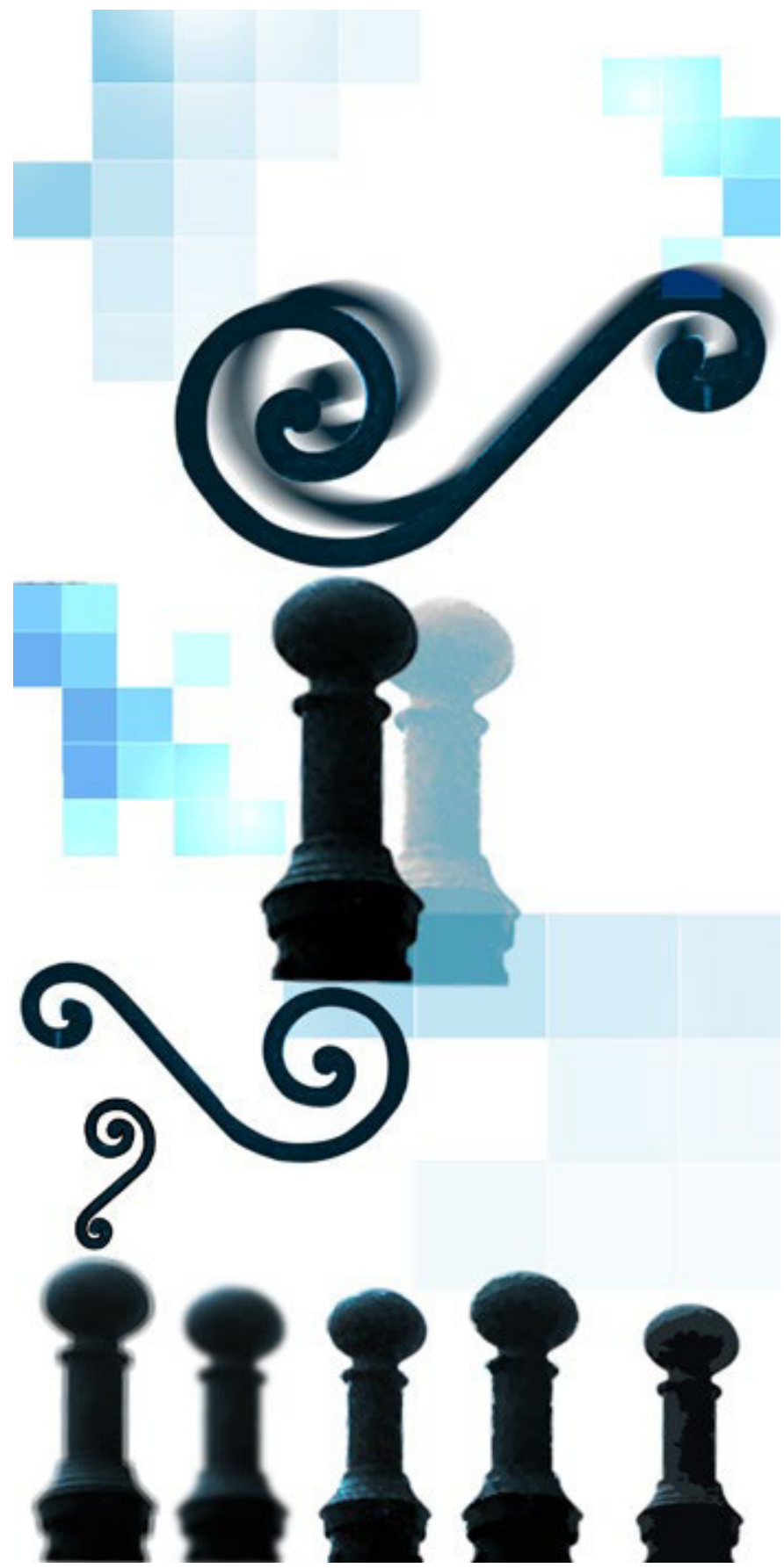

\section{Introducción}

l derecho a la educación es un ideario fundamental en - las sociedades modernas; sin ser considerado como más importante que otros, es pilar fundamental para el desarrollo de las sociedades, al ser un mecanismo que promueve la formación de individuos y ciudadanos. La educación genera desarrollo humano, a nivel personal y social, desde el reconocimiento de la historia propia en relación con el mundo; genera libertad y responsabilidad frente al cuidado del planeta; promueve respeto propio y hacia los otros y es motor del desarrollo económico. Así, los demás derechos dependen de la garantía del derecho a la educación y, en tal sentido, ésta se convierte en un derecho multiplicador que debe ser garantizado por las naciones (ONU, 1999).

La Declaración Universal de Derechos Humanos establece que la educación es un derecho universal y que, en la etapa elemental, debe ser gratuita y obligatoria, además de accesible en los niveles técnico y profesional. El derecho a la educación apoya procesos humanos relevantes, como el desarrollo pleno de las capacidades individuales, la vida y el trabajo digno, la calidad de vida, la toma de decisiones informadas y el aprendizaje.

Sin embargo, la Declaración Mundial sobre Educación para Todos (UNESCO, 1990) reconoce que aún no se ha asegurado el acceso universal a la primaria ni la conclusión del ciclo de educación básico; subraya que la plena realización del Derecho a la Educación no es solo una cuestión de acceso (UNESCO 2008). De la mano con tales reflexiones, se tomó la decisión de adoptar algunas metas próximas, entre ellas: ampliar la protección y educación de la primera infancia; establecer una enseñanza primaria gratuita y obligatoria para todos; promover el aprendizaje y la preparación para la vida activa para jóvenes y adultos; alcanzar la paridad de los sexos en el año 2005 y la igualdad entre sexos para 2015 y mejorar la calidad educativa de la educación. Así, este enfoque de garantía del derecho a la educación, basado en los Derechos Humanos, abarca desde el acceso hasta la calidad y la relación con el entorno familiar, escolar y social de los estudiantes.

Son múltiples las iniciativas sobre estrategias para el seguimiento a la materialización del derecho a la educación; sin embargo, 


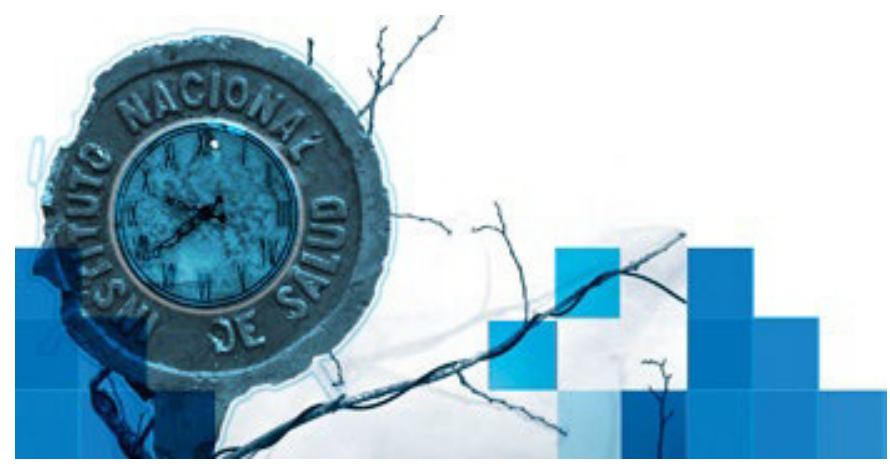

éstas pueden presentar algunas dificultades: 1) Debilidad entre la relación del fundamento conceptual sobre cómo se entiende el derecho a la educación y los indicadores que se miden; 2) Poco consenso entre los indicadores que dan cuenta del derecho a la educación; 3) Escasa información o difícil acceso a indicadores claves en la medición del derecho a la educación. Dicho esto, la presente investigación aporta al escenario, en la medida que: 1) A partir de un marco sobre el cual se fundamenta el derecho a la educación, selecciona variables pertinentes y de fácil acceso. 2) Implementa la metodología Delphi para llevar a cabo un proceso de validación, de tal manera que sea posible el consenso entre expertos sobre los indicadores que componen cada una de las dimensiones del Índice del Derecho a la Educación.

\section{La educación como derecho: El marco conceptual de las 4 Aes}

En su publicación de 2004 Tomasevski propuso un marco conceptual para hacer seguimiento a los avances en la implementación del derecho a la educación. Su objetivo era traducir los estándares aceptados, y promulgados en los acuerdos internacionales sobre derechos de la niñez, a una guía concreta de referencia para la implementación y seguimiento de avance hacia su implementación. Para lograr esto, primero hizo un inventario de los estándares relacionados con los Derechos Humanos consignados en compromisos globales y relevantes a la educación, para luego proponer cómo se traducen en la práctica, en el diseño, implementación y seguimiento de las políticas públicas. Finalmente, lo que se quiere lograr es volver operativa la visión de la educación basada en los derechos y extenderla al ámbito de lo práctico.

El aporte fundamental del marco es la tipología para clasificar las obligaciones legales de los Estados -que se derivan de los derechos promulgados en el marco normativo-, en cuatro grandes categorías o dimensiones: 1) Disponibilidad (o asequibilidad); 2) Aceptabilidad; 3) Accesibilidad y 4) Adaptabilidad. Esta propuesta busca que, al traducir el universo de compromisos a un número de dimensiones manejable -solo cuatro-, sea posible definir indicadores que sirvan para cuantificar los avances logrados en la implementación del derecho a la educación en general y, en particular, en cada una de las categorías definidas de obligaciones adquiridas (Tomasevski, 2004). Las dimensiones se definen de la siguiente manera:

1. Disponibilidad (Asequibilididad). Hace referencia a las obligaciones del Estado en cuanto al derecho a la educación. Como derecho civil y político, el Estado debe garantizar la admisión a instituciones educativas que respeten la libertad de educación. Como derecho social y económico, el Estado debe garantizar la existencia de educación gratuita y obligatoria para todos los niños en edad escolar. Como derecho cultural, debe garantizar el respeto a la diversidad, a través de los derechos a las minorías.

2. Aceptabilidad. Se trata del esfuerzo por garantizar la calidad de la educación. El Estado debe establecer, controlar y exigir estándares de calidad, tanto para las instituciones públicas como privadas. En esta categoría se incluyen compromisos en el currículo, los materiales, la capacitación docente y el lenguaje de instrucción, entre otros, que estén en armonía con las habilidades y necesidades de los estudiantes. Así mismo, el Derecho a la Educación ha extendido las fronteras de aceptabilidad, examinando y modificando los programas educativos, los textos y los métodos de enseñanza-aprendizaje, con el fin de volver la educación aceptable para todos.

3. Accesibilidad. Hace referencia a que el derecho a la educación debe realizarse progresivamente, asegurando la educación gratuita, obligatoria e inclusiva a todos los niños en edad escolar, y facilitando, en la medida en que sea posible, el acceso a la educación post-obligatoria, es decir, la educación media y superior.

\section{La escuela entonces, se considera un territorio, no por todo lo que la rodea o por entenderla como contenedor de cosas, sino por ser una construcción permanente y no acabada... 99}


4. Adaptabilidad. Se refiere a la capacidad de adaptar las escuelas a los niños, revocando la tradición de forzarles a adecuarse a las condiciones de la escuela. Se busca garantizar el cumplimiento de los Derechos Humanos en la educación, adaptándola a ellos progresivamente. En efecto, el derecho internacional de los Derechos Humanos establece que la educación es su principal mecanismo de promoción.

\section{Iniciativas de índices del derecho a la educación}

Existen diversas iniciativas que buscan, al igual que el IDE, sintetizar indicadores para medir el avance en el cumplimiento del derecho a la educación. A continuación se enseñan las más cercanas al IDE para Colombia, pero antes se debe ahondar en el sentido del IDE. Con el fin de medir los avances de los países frente al programa de Educación Para Todos (EPT), la UNESCO adoptó el Índice de Desarrollo de la Educación para todos (IDE), índice compuesto que da cuenta de cuatro objetivos de EPT: 1) La enseñanza primaria universal (objetivo 2), medida por la tasa neta de escolarización ajustada en primaria; 2) La alfabetización de adultos (primera parte, objetivo 4), medida por la tasa de alfabetización de las personas de 15 años y más; 3) La paridad e igualdad entre los sexos (objetivo 5), medida por el promedio de los índices de paridad entre los sexos de las tasas brutas de escolarización en la enseñanza primaria y secundaria, y de la tasa de alfabetización de los adultos; 4) La calidad de la educación (objetivo 6), medida por la tasa de supervivencia en el quinto grado de primaria (UNESCO, 2011). La imposibilidad de tener más información impide incluir en este índice más variables, lo que constituye una debilidad importante. Sin embargo, cada uno de los componentes del índice tiene una métrica coherente.

Por su parte, la fundación IDEA (s.f.) construyó el índice de Calidad Educativa (ICE) para México, como herramienta para dar información y contribuir a la discusión de política educativa. El índice fue estimado empleando metodologías econométricas donde la variable dependiente son los puntajes de las pruebas estandarizadas aplicadas por el gobierno, y las variables independientes están relacionadas con el maestro, las entidades educativas y los elementos pedagógicos. Aunque es un aporte interesante, es complicado inferir elementos relacionados con el derecho a la educación; además, este tipo de metodología puede invitar a discusiones técnicas complejas.

Desde otra perspectiva, la Unicef (2005) desarrolló tres índices de los derechos de la niñez, uno por cada grupo de edades: 0 a 5 años, 6 a 11 años y 12 a 17 años. Los indicadores utilizados miden el grado de cumplimiento de tres derechos fundamentales para cada grupo, dos de los cuales son comunes a los tres grupos de edades: derecho a la vida y derecho a la educación. Para los niños de 0 a 5 años el tercero es el derecho a crecer saludable y bien nutrido; para los niños de 6 a 11 años es el derecho a vivir sin maltrato ni violencia. En cuanto a los niños de 12 a 17 años, el tercer derecho es a la no explotación laboral.

El derecho a la educación está compuesto, para el grupo de 0 a 5 años, por dos indicadores, el primero es Mujeres analfabetas, que hace referencia al porcentaje de mujeres de 15 años o más que no saben leer. El segundo es inasistencia y hace referencia al número de niños de 5 años que no están matriculados. Para el grupo de 6 a 11 años la dimensión sobre el derecho a la educación está compuesta por: Inasistencia escolar, rezago escolar y porcentaje de niños que ingresan a primaria y no terminan. Finalmente, para el grupo de 12 a 17 años esta dimensión se compone por: Inasistencia escolar, rezago escolar y porcentaje de niños que ingresan a secundaria y no terminan. En la dimensión del derecho a la educación este índice no contempla elemento de calidad ni equidad.

Para el caso colombiano, la Secretaría de Educación del Distrito (2009) presentó un análisis del índice del derecho a la educación que sigue conceptualmente el estudio de Tomasevski (2004) sobre las cuatro Aes, sin embargo, la interpretación e indicadores empleados no responden adecuadamente con la metodología. Al tiempo, la Defensoría del Pueblo (2004) concretó un extenso trabajo (Sistema de Seguimiento y Evaluación de la Política Pública) que gira alrededor del derecho a la educación y plantea una batería de indicadores altamente relacionados con lo definido mientras, al tiempo, presenta una serie de instrumentos relevantes para entender las dinámicas sobre la materialización del derecho a la educación. A pesar de ello, tanto los instrumentos como los indicadores requieren de la voluntad de las entidades para diligenciar los instrumentos y suministrar la información, lo que hace difícil su implementación.

La presente investigación aporta a esta literatura en dos aspectos: Hace una selección inicial juiciosa de los indicadores que dan cuenta del derecho a la educación; y concreta un proceso de
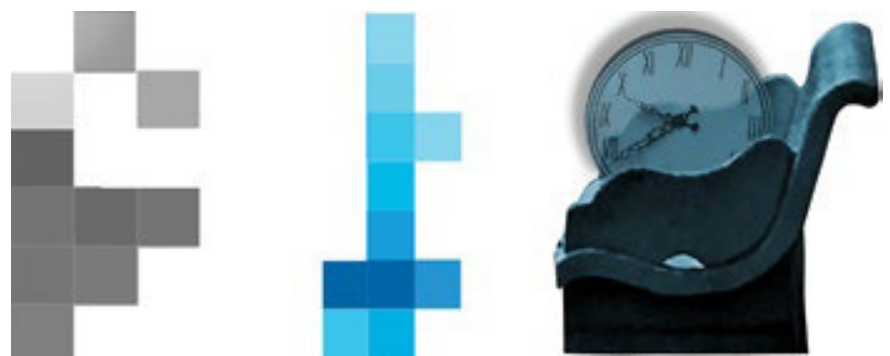
validación de expertos que permite asegurar que esos indicadores se clasifican en las dimensiones correctas.

\section{Propuesta de indicadores}

El criterio para la selección inicial de los indicadores para la construcción del IDE es la disponibilidad y calidad de datos oficiales. En particular, los datos deben ser confiables y tener la frecuencia y representatividad a nivel geográfico y de las poblaciones de interés. La metodología para construir los índices combina los criterios de 1) Robustez técnica y 2) Simplicidad para su comunicación e interpretación por parte del público no especializado. En esta medida, se reconoce que para avanzar en el cumplimiento del derecho a la educación, es necesario hacer visible ante la opinión pública la situación actual, para luego hacer seguimiento a los avances y retrocesos. De acuerdo con ello, la presente investigación propone una taxonomía que agrupa 85 subindicadores en 12 indicadores globales.

1. Tasa de cobertura neta. Porcentaje de estudiantes matriculados en un nivel educativo que tienen la edad teórica para cursar este nivel; cuenta con 4 sub-indicadores, uno por cada nivel educativo.

2. Relación alumno-docente en el sector oficial. Número de estudiantes que en promedio tiene a cargo un profesor; el indicador no tiene sub-indicadores.

3. Índice de paridad de género en cobertura. Relación entre el valor de la tasa de cobertura neta para el sexo femenino y el valor del mismo indicador para el sexo masculino; el indicador no tiene sub-indicadores.

4. Tasa de extra-edad. Porcentaje de alumnos matriculados en un grado escolar cuya edad supera en dos años o más la edad teórica para cursar el grado. Cuenta con 5 sub-indicadores, uno por cada nivel educativo y uno más que evalúa la brecha de extra-edad por sector (oficial o no oficial).

5. Nivel educativo de los docentes. Porcentaje de docentes que tienen educación superior (mínimo pregrado: licenciatura u otras profesiones). También se tiene en cuenta el porcentaje de profesores con posgrado; cuenta con 4 sub-indicadores, correspondientes a la combinación entre la zona (rural o urbana) y el nivel de estudios (profesional o posgrado).

6. Porcentaje de la matrícula en jornada única en el sector oficial. Porcentaje de la población estudiantil en el sector oficial que está en jornada única; cuenta con 2 sub-indicadores, uno para cada sector (oficial y no oficial).
7. Tasa de deserción intra-anual del sector oficial. Porcentaje de estudiantes que salen del sistema educativo formal; el indicador no tiene sub-indicadores.

8. Tasa de reprobación en el sector oficial. Porcentaje de estudiantes que no aprobaron el año escolar; el indicador no tiene sub-indicadores.

9. Tasa de terminación de educación primaria y secundaria por una cohorte. Porcentaje de estudiantes que finaliza con éxito su proceso formativo (por nivel de formación). Tiene 2 sub-indicadores: uno para educación primaria y otro para secundaria.

10. Tránsito inmediato entre niveles de educación. Porcentaje de estudiantes que hacen un tránsito inmediato entre el último grado de un nivel y el primer grado del nivel siguiente. Tiene 4 sub-indicadores, correspondientes al tránsito entre los niveles educativos.

11. Esperanza de vida escolar del sector oficial.Promedio del número de años que tarda un estudiante en el sistema educativo oficial (primaria y secundaria); el indicador no tiene sub-indicadores.

12. Porcentaje de colegios con el mínimo deseado en los resultados de las pruebas Saber $3^{\circ}, 5^{\circ}, 9^{\circ}$ y $11^{\circ}$. Porcentaje de colegios que obtuvieron al menos el mínimo desempeño deseado en las pruebas Saber. Tiene 16 sub-indicadores, correspondientes a la combinación entre grado $\left(3^{\circ}, 5^{\circ}, 9^{\circ}\right.$ o $\left.11^{\circ}\right)$, zona (rural o urbana) y sector (oficial o no oficial).

\section{Metodología}

La clasificación de los indicadores se realizó siguiendo el método Delphi, en el cual se indaga a expertos, a través de varias rondas, interrogando por una posición frente a un tema o un problema, con el fin de llegar a consensos. Su uso es adecuado en contextos particulares que presentan retos específicos. En particular, es apropiado cuando se cuenta con las siguientes condiciones:

1. El problema no se beneficiaría tanto de técnicas analíticas tradicionales, sino que requiere de considerar juicios subjetivos de colectivos o grupos de personas.

2. Se trata de un problema complejo que tradicionalmente no ha sido abordado con las personas que cuentan con la experticia o experiencia requeridas para entenderlo.

3. Se requieren consensos entre un grupo mayor de individuos, superior al necesario en sesiones típicas de grupos focales.

4. El tiempo y el costo hacen difícil conducir varios grupos focales. 
5. La eficiencia de los grupos focales se puede mejorar incorporando otros procesos de comunicación entre los participantes.

6. El anonimato es deseable, porque se necesita conocer opiniones divergentes o hay implicaciones políticas de difícil conciliación; también porque se quiere tener en cuenta el punto de vista de participantes heterogéneos pero evitar el fenómeno de la deseabilidad social (Slocum, 2003).

Las características más importantes de este método son: 1) La participación de los expertos es anónima, para evitar que quienes posean mayor prestigio en el área o quienes sean más participativos dominen las sesiones; 2) Antes de cada ronda se da información sobre la ronda anterior; 3) Puede hacerse con un número grande de participantes y de manera no presencial. El método se ha conducido tradicionalmente por correo electrónico, pero se pueden realizar variaciones con sesiones presenciales o en línea (Slocum, 2003).
En educación, el método Delphi ha sido utilizado en procesos de autoevaluación para mejorar la didáctica de las ciencias naturales (Charro, Charro y Niño, 2013), de las ciencias sociales (Pozo, Gutiérrez y Rodríguez, 2007) y de programas de formación a distancia (García, Aquino, Guzmán y Medina, 2012); detectar tendencias en educación a distancia (López y Bañuls, 2017); desarrollar un inventario de medición para la evaluación pedagógica (Elmendorf y Song, 2015); o para desarrollar un marco de competencias para los profesores de secundaria de matemáticas (Muñiz, Alonso, Rodríguez y Valcke, 2017). La implementación del método se realizó mediante una página Web diseñada para este fin. El proceso de validación se realizó entre el 21 de marzo y el 7 de abril de 2017, tiempo en el cual se realizaron dos rondas. En la primera se presentó a cada experto los doce indicadores con una breve explicación de qué mide y cómo se mide. Con base en esta información, los expertos debían seleccionar la dimensión que consideraran más apropiada para cada uno de los indicadores. La Figura 1 expone la interfaz de la herramienta en la primera ronda.

Figura 1. Funcionamiento de la página ide-validacion.org

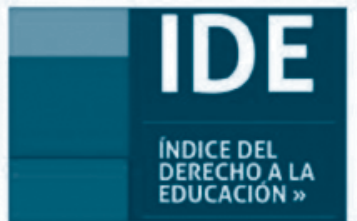

Ronda 1 - (Indicador 1 de 12)

\section{Tasa de cobertura neta}

\section{Cómo se mide:}

(Matricula en el nivel educativo n que tiene la edad

oficial e para cursarlo/Población con la edad teórica e

del nivel educativo) $* 100$

\begin{abstract}
matriculados en un nivel educativo que tienen la edad
\end{abstract}
teórica para cursarlo y el total de la población

correspondiente a esa misma edad.

Respuesta a factores que limitan el acceso a la Educación.

\section{Disponibilidad}

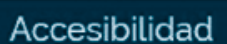

Adaptabilidad

\section{Aceptabilidad}

Nota. Los expertos seleccionan la dimensión que consideran apropiada para cada indicador 
Al terminar la ronda se asigna un indicador a una dimensión, si ésta obtiene un porcentaje de votos superior al $65 \%$. De no cumplirse el requisito se inicia una nueva ronda y se presentan nuevamente a los expertos aquellos indicadores sin clasificar. En la nueva ronda los expertos cuentan con información adicional, correspondiente a las estadísticas agregadas que resultaron de la participación general en la ronda anterior y que se resumen en una gráfica de barras en la nueva página de clasificación del indicador. Adicionalmente, se abrió un espacio para recolectar los comentarios de los participantes sobre cada indicador, su forma de medición, o las consideraciones que deberían de tenerse en cuenta durante las fases posteriores del IDE.

\section{Participantes}

Siguiendo las recomendaciones de Hsu y Sandford (2007), es importante considerar: tomadores de decisión, quienes utilizarán los resultados del estudio Delphi; profesionales y miembros de los equipos de trabajo involucrados con el problema y con la toma de decisiones; y personas que otros denominan líderes. Con esto en mente, los expertos invitados fueron profesores, académicos, tomadores de decisión y empresarios interesados en mejorar la calidad de la educación, todos con un profundo conocimiento del sistema educativo y con sentido de urgencia por la materialización de la educación como derecho fundamental.

Los expertos invitados fueron propuestos por representantes de cada una de las organizaciones que conforman la Alianza Educación Compromiso de Todos, con base en el trabajo que éstas desarrollan en pro de la educación. A partir de esto, se construyó una base de datos compuesta, entre otros, por 851 expertos vinculados a instituciones de educación, ONGs, secretarías de educación e instituciones gubernamentales. Se envió un correo de invitación para participar en esta validación a cada uno de los expertos; de ellos, 36 fueron devueltos, 223 expertos confirmaron su participación y 158 participaron en una de las dos rondas.

Tabla 1. Estadísticas descriptivas de los expertos participantes

\begin{tabular}{|c|c|c|c|c|}
\hline & Promedio & $\begin{array}{c}\text { Desviación } \\
\text { Estándar }\end{array}$ & $\mathbf{n}$ & $\%$ \\
\hline Edad & 45.5 & 11.37 & & \\
\hline Experiencia en el sector educativo (años) & 19.4 & 10.9 & & \\
\hline Público & 13.4 & 11.5 & & \\
\hline Privado & 8.3 & 8.1 & & \\
\hline \multicolumn{5}{|l|}{ Sexo } \\
\hline Femenino & & & 87 & $55.1 \%$ \\
\hline Masculino & & & 71 & $44.9 \%$ \\
\hline \multicolumn{5}{|l|}{ Ciudad } \\
\hline Bogotá & & & 96 & $60.8 \%$ \\
\hline Otros & & & 62 & $39.2 \%$ \\
\hline \multicolumn{5}{|l|}{ Tipo de organización } \\
\hline Institución de Educación Básica y Media & & & 50 & $31.8 \%$ \\
\hline Organización sin ánimo de lucro (Nacional) & & & 30 & $19.1 \%$ \\
\hline Secretaría de Educación & & & 30 & $19.1 \%$ \\
\hline Institución de Educación Superior & & & 21 & $13.4 \%$ \\
\hline Otra & & & 10 & $6.4 \%$ \\
\hline Organización sin ánimo de lucro (Internacional) & & & 8 & $5.1 \%$ \\
\hline Sector empresarial & & & 5 & $3.2 \%$ \\
\hline Entidad pública del orden nacional & & & 3 & $1.9 \%$ \\
\hline \multicolumn{5}{|l|}{ Cargo } \\
\hline Directivo & & & 54 & $34.4 \%$ \\
\hline Profesor & & & 46 & $29.3 \%$ \\
\hline Profesor e investigador & & & 22 & $14.0 \%$ \\
\hline Otra & & & 11 & $7.0 \%$ \\
\hline Profesional o técnico & & & 11 & $7.0 \%$ \\
\hline Asesor & & & 5 & $3.2 \%$ \\
\hline Investigador & & & 5 & $3.2 \%$ \\
\hline Consultor & & & 3 & $1.9 \%$ \\
\hline
\end{tabular}


La Tabla 1 presenta las estadísticas descriptivas. Los expertos participantes cuentan con una experiencia importante en el sector educativo, cerca de 19 años en promedio, tiempo en el cual han estado vinculados principalmente al sector público. En su mayoría, los participantes son mujeres (55.1\%), están vinculados como docentes o directivos (77.7\%), especialmente en instituciones de educación básica y media, organizaciones sin ánimo de lucro o secretarías de educación (70.1\%) y residen en Bogotá $(60.8 \%)$. De quienes residen fuera de Bogotá hay representación de 25 departamentos de Colombia y cuatro países extranjeros. La Figura 2 permite ver la distribución de los expertos que residen en Colombia (fuera de Bogotá); la mayoría habita las regiones occidentales: Andina, Caribe y Pacífico.

Figura 2. Distribución de los expertos que residen en Colombia (fuera de Bogotá)

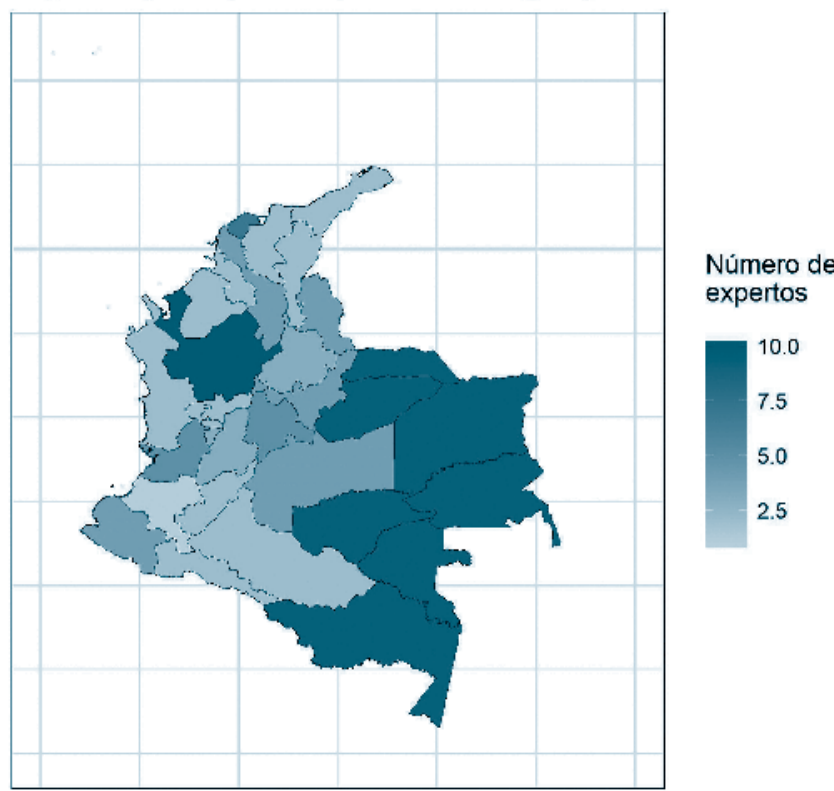

\section{Resultados}

En la primera ronda participaron 131 expertos, de los cuales 124 realizaron la clasificación completa de los doce indicadores. Al terminar esta ronda se llegó a un consenso en el indicador Porcentaje de colegios con el mínimo deseado en los resultados de las pruebas Saber $3^{\circ}, 5^{\circ}, 9^{\circ}$ y $11^{\circ}$, que fue asignado a la dimensión Aceptabilidad (75.4\%). Otros indicadores tuvieron una dimensión de más del $50 \%$ de los votos, pero se mantuvieron en la segunda ronda, al no superar el criterio de $65 \%$; estos fueron: Índice de Paridad de Género en Cobertura (56.1\%), Nivel educativo de los docentes (55.3\%), Tasa de deserción intra-anual del sector oficial (54.8\%), Relación alumno-docente (sector oficial) (54.0\%), Tasa extraedad y Tasa de cobertura neta $(51.2 \%)$.

En general, de acuerdo con la selección de los expertos, es posible establecer tres grupos de indicadores. En el primero se observa la preferencia por una dimensión y una decisión dividida entre las demás (Relación alumno-docente (sector oficial), Índice de Paridad de Género en Cobertura, Tasa de deserción intra-anual del sector oficial, Porcentaje de colegios con el mínimo deseado en los resultados de las pruebas Saber $3^{\circ}, 5^{\circ}, 9^{\circ}$ y $11^{\circ}$ ). En el segundo grupo hay dos dimensiones que sobresalen (Tasa de cobertura neta, Tasa extraedad, Nivel educativo de los docentes, Tasa de reprobación en el sector oficial, Tasa de terminación de educación primaria y secundaria por una cohorte, y Esperanza de vida escolar del sector oficial).

En el último grupo las votaciones estuvieron repartidas entre las cuatro dimensiones; para el indicador Porcentaje de la matrícula en jornada única en el sector oficial, los porcentajes de votación se encuentran entre $17.1 \%$ y $38.2 \%$, mientras que en Tránsito inmediato entre Niveles de educación estuvieron entre $15.6 \%$ y $31.1 \%$. La Figura 3 expone el porcentaje de expertos que seleccionó cada dimensión para cada uno de los indicadores. La línea punteada, ubicada en $65 \%$, es el criterio de elección del indicador para la primera ronda. 
Figura 3. Clasificación de indicadores en la primera ronda. La línea roja muestra el criterio de elección para esta ronda

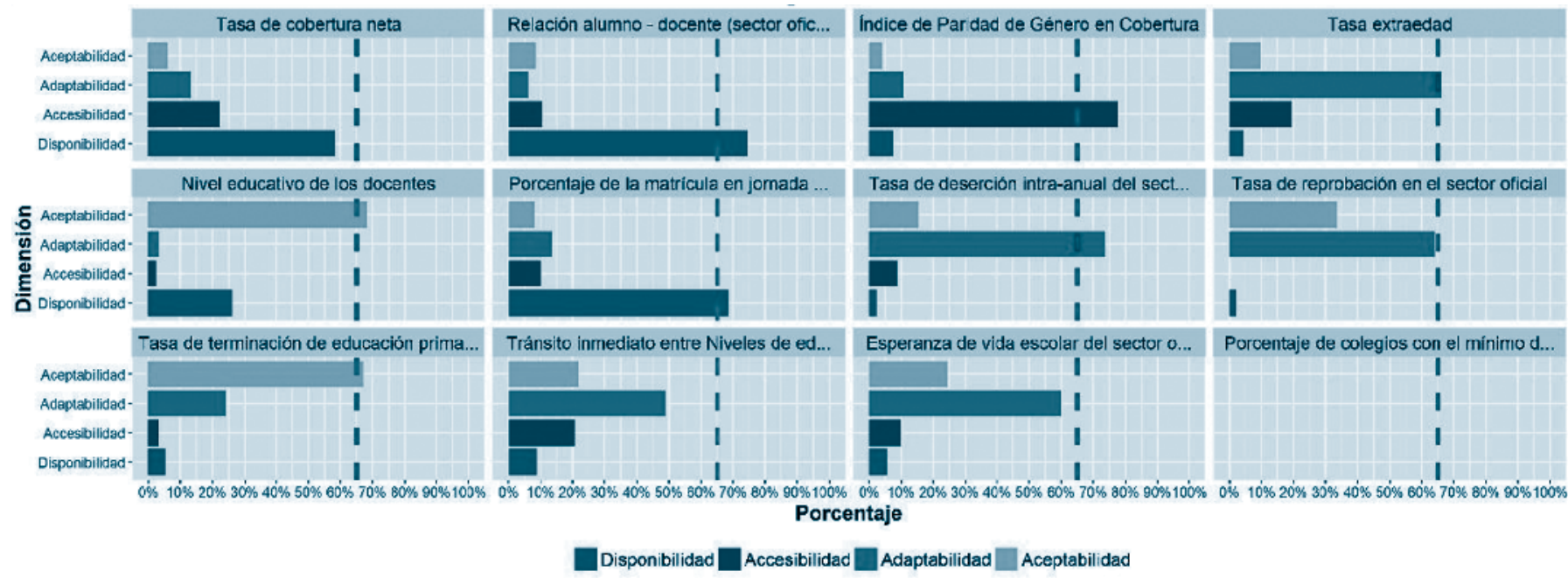

En la segunda ronda participaron 102 expertos, 85 realizaron la clasificación de los once indicadores disponibles. Al terminar, siete de los indicadores superaron el umbral y fueron asignados a la dimensión escogida por los expertos, estos fueron: Relación alumno-docente (sector oficial), Índice de Paridad de Género en Cobertura, Tasa extraedad, Nivel educativo de los docentes,
Porcentaje de la matrícula en jornada única en el sector oficial, Tasa de deserción intra-anual del sector oficial, Tasa de terminación de educación primaria y secundaria por una cohorte. En estos casos se consolidó la intención de los expertos, expresada en los resultados de la primera ronda. La Figura 4 presenta los resultados.

Figura 4. Clasificación de indicadores en la segunda ronda

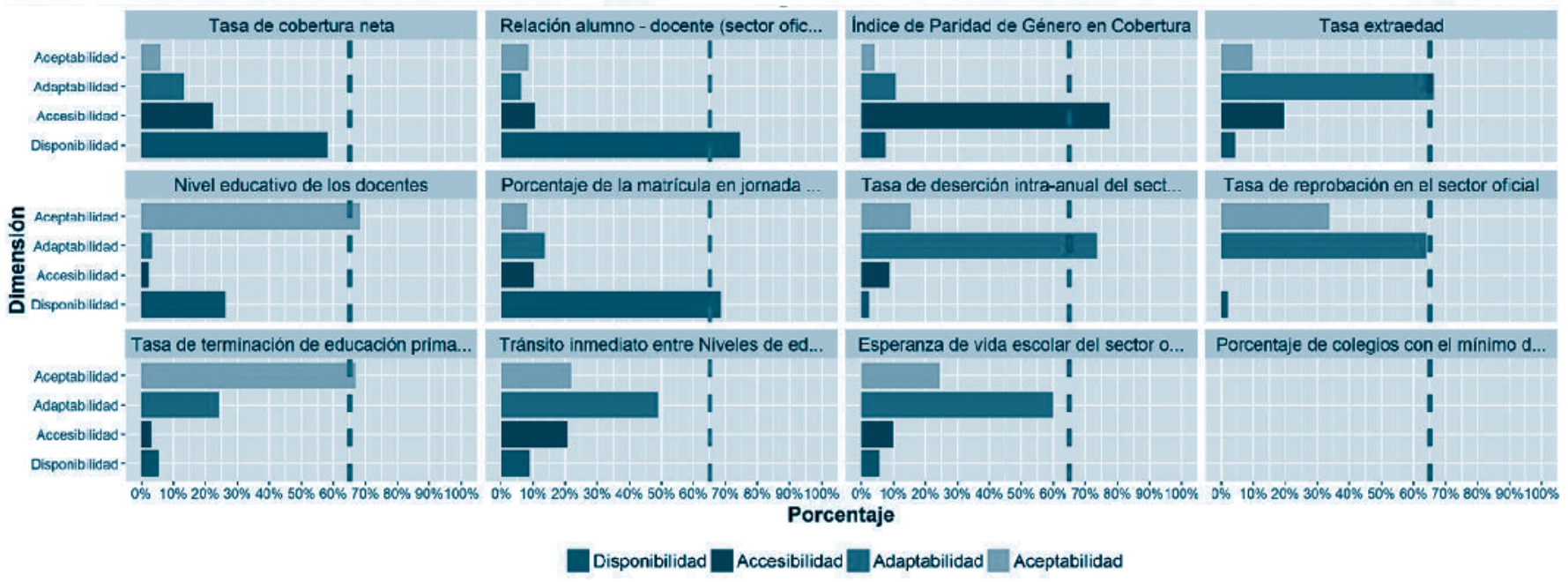


En los cuatro indicadores restantes no se llegó a un acuerdo que superara el umbral del $65 \%$. Sin embargo, en tres de estos cuatro el porcentaje de votos de la primera opción superó el 55\%: Tasa de cobertura neta (58.2\%), Esperanza de vida escolar del sector oficial (60.0\%), y Tasa de reprobación en el sector oficial (64.1\%). El cuarto indicador, Tránsito inmediato entre Niveles de educación, tuvo la votación más cerrada de la ronda, la primera opción obtuvo el $48.9 \%$ de los votos, aunque fue más del doble que la segunda (21.7\%).
Teniendo en cuenta estos resultados, se decidió realizar un análisis estadístico. Primero se usó una prueba $\chi 2$ para evaluar si la proporción de expertos que seleccionó una dimensión, en un determinado indicador, es la misma para todas las dimensiones, concluyendo, con un nivel alfa de 0.001 , que hay diferencias entre las proporciones de cada dimensión. Los resultados se presentan en la Tabla 2.

Tabla 2. Prueba $\chi 2$ para proporciones en la segunda ronda

\begin{tabular}{|c|c|c|c|c|c|}
\hline Indicador & Disponibilidad & Accesibilidad & Adaptabilidad & Aceptabilidad & p-valor \\
\hline 1 & 57 & 22 & 13 & 6 & $<0.001$ \\
\hline 2 & 70 & 10 & 6 & 8 & $<0.001$ \\
\hline 3 & 7 & 73 & 10 & 4 & $<0.001$ \\
\hline 4 & 4 & 18 & 61 & 9 & $<0.001$ \\
\hline 5 & 23 & 2 & 3 & 60 & $<0.001$ \\
\hline 6 & 61 & 9 & 12 & 7 & $<0.001$ \\
\hline 7 & 2 & 8 & 67 & 14 & $<0.001$ \\
\hline 8 & 2 & 0 & 59 & 31 & $<0.001$ \\
\hline 9 & 5 & 3 & 22 & 61 & $<0.001$ \\
\hline 10 & 8 & 19 & 45 & 20 & $<0.001$ \\
\hline 11 & 5 & 9 & 54 & 22 & $<0.001$ \\
\hline
\end{tabular}

Posteriormente, se usó una prueba $\mathrm{t}$ de una muestra para comparar las proporciones y determinar si existe una diferencia, estadísticamente significativa, entre la opción que fue seleccionada por un mayor número de expertos y las demás. Específicamente, el análisis se centra en la comparación de las proporciones entre las dos opciones más votadas. La Tabla 3 presenta los resultados del análisis, donde se puede observar que el resultado de la prueba t es estadísticamente significativo en todos los indicadores de la segunda ronda. En estos casos se puede concluir que el porcentaje de expertos que seleccionó la primera opción es mayor que el que seleccionó la segunda. 
Tabla 3. Prueba t para proporciones por rondas

\begin{tabular}{|c|c|c|c|c|c|c|c|c|}
\hline & \multicolumn{4}{|c|}{ Ronda 1} & \multicolumn{4}{|c|}{ Ronda 2} \\
\hline Indicador & $\begin{array}{c}\text { Primera } \\
\text { opción }\end{array}$ & $\begin{array}{c}\text { Segunda } \\
\text { opción }\end{array}$ & $\begin{array}{c}\text { Participación } \\
\text { total }\end{array}$ & p-valor & $\begin{array}{l}\text { Primera } \\
\text { opción }\end{array}$ & $\begin{array}{c}\text { Segunda } \\
\text { opción }\end{array}$ & $\begin{array}{c}\text { Participación } \\
\text { total }\end{array}$ & p-valor \\
\hline 1 & 65 & 41 & 127 & $<0.01$ & 57 & 22 & 98 & $<0.01$ \\
\hline 2 & 67 & 21 & 124 & $<0.01$ & 70 & 10 & 94 & $<0.01$ \\
\hline 3 & 69 & 24 & 123 & $<0.01$ & 73 & 10 & 94 & $<0.01$ \\
\hline 4 & 63 & 36 & 123 & $<0.01$ & 61 & 18 & 92 & $<0.01$ \\
\hline 5 & 68 & 39 & 123 & $<0.01$ & 60 & 23 & 88 & $<0.01$ \\
\hline 6 & 47 & 31 & 123 & 0.001 & 61 & 12 & 89 & $<0.01$ \\
\hline 7 & 68 & 29 & 124 & $<0.01$ & 67 & 14 & 91 & $<0.01$ \\
\hline 8 & 54 & 49 & 123 & 0.204 & 59 & 31 & 92 & $<0.01$ \\
\hline 9 & 57 & 39 & 122 & $<0.01$ & 61 & 22 & 91 & $<0.01$ \\
\hline 10 & 38 & 37 & 122 & 0.461 & 45 & 20 & 92 & $<0.01$ \\
\hline 11 & 51 & 36 & 122 & 0.002 & 54 & 22 & 90 & $<0.01$ \\
\hline 12 & 95 & 15 & 126 & $<0.01$ & & & & \\
\hline
\end{tabular}

Con base en estos resultados, los indicadores fueron clasificados al terminar esta ronda. La clasificación puede verse en la Tabla 4.

Tabla 4. Clasificación de los indicadores en las cuatro dimensiones

\begin{tabular}{|l|l|}
\hline Indicador & \multicolumn{1}{|c|}{ Dimensión } \\
\hline Tasa de cobertura neta & Disponibilidad \\
Porcentaje de la matrícula en jornada única en el sector oficial & Disponibilidad \\
\hline Índice de paridad de género en cobertura & Accesibilidad \\
\hline Tasa extraedad & Adaptabilidad \\
Tasa de deserción intra-anual del sector oficial & Adaptabilidad \\
Tasa de reprobación en el sector oficial & Adaptabilidad \\
Tránsito inmediato entre niveles de educación & Adaptabilidad \\
Esperanza de vida escolar del sector oficial & Adaptabilidad \\
\hline $\begin{array}{l}\text { Nivel educativo de los docentes } \\
\text { Tasa de terminación de educación primaria y secundaria por una cohorte } \\
\text { Porcentaje de colegios con el mínimo deseado en los resultados de las pruebas } \\
\text { Saber } 3^{\circ}, 5^{\circ}, 9^{\circ} \text { y } 11^{\circ}\end{array}$ & Aceptabilidad \\
\hline
\end{tabular}


En la segunda ronda se consolidaron las opciones más votadas durante la primera vuelta; no obstante, la elección de los expertos transitó entre las diferentes dimensiones. En efecto, algunos que habían elegido la opción más votada en la primera ronda cambiaron su voto en la segunda. También hubo expertos que mantuvieron su voto a pesar de que su opción fue la menos votada. La Tabla 4 expone las matrices de transición de las decisiones de los expertos para cada uno de los indicadores.
Por ejemplo, para el indicador Índice de paridad de género en cobertura, se observa que el $80 \%$ de los expertos que le clasificaron en la primera ronda en Disponibilidad, para la segunda ronda lo hicieron en Accesibilidad; $62 \%$ de quienes habían clasificado este indicador en Adaptabilidad durante la primera ronda, lo clasificaron en Accesibilidad en la segunda, y $75 \%$ de los que le ubicaron en Aceptabilidad en la primera ronda, cambió su decisión por Accesibilidad. Los cuadros de la Tabla 5 se leen de manera similar. Adicionalmente, el porcentaje de cambio se ve reflejado en la intensidad del color.

Tabla 5. Matriz de transición de las decisiones de los expertos

\begin{tabular}{cc|c|c|c|}
\multicolumn{4}{c}{ Tasa de cobertura neta } \\
\hline \multirow{2}{*}{ Disponibilidad } & $71 \%$ & $12 \%$ & $12 \%$ & $5 \%$ \\
\hline Accesibilidad & $55 \%$ & $36 \%$ & $9 \%$ & $0 \%$ \\
\hline Adaptabilidad & $40 \%$ & $20 \%$ & $40 \%$ & $0 \%$ \\
\hline Aceptabilidad & $25 \%$ & $25 \%$ & $0 \%$ & $50 \%$ \\
\hline
\end{tabular}

\begin{tabular}{c|c|c|c|c|} 
& \multicolumn{4}{c}{ Tasa extraedad } \\
\cline { 2 - 5 } & \multicolumn{1}{c}{ D } & Acc & Ad & Ace \\
\cline { 2 - 5 } Disponibilidad & $11 \%$ & $33 \%$ & $56 \%$ & $0 \%$ \\
\hline Accesibilidad & $5 \%$ & $38 \%$ & $52 \%$ & $5 \%$ \\
\hline Adaptabilidad & $3 \%$ & $8 \%$ & $78 \%$ & $11 \%$ \\
\hline Aceptabilidad & $0 \%$ & $20 \%$ & $60 \%$ & $20 \%$ \\
\hline
\end{tabular}

\begin{tabular}{c|c|c|c|c|} 
& \multicolumn{4}{c}{$\begin{array}{c}\text { Esperanza de vida escolar del } \\
\text { sector oficial }\end{array}$} \\
\cline { 2 - 5 } & D & Acc & Ad & Ace \\
\cline { 2 - 5 } Disponibilidad & $25 \%$ & $25 \%$ & $25 \%$ & $25 \%$ \\
\hline Accesibilidad & $0 \%$ & $0 \%$ & $100 \%$ & $0 \%$ \\
\hline Adaptabilidad & $9 \%$ & $9 \%$ & $62 \%$ & $21 \%$ \\
\hline Aceptabilidad & $0 \%$ & $13 \%$ & $40 \%$ & $47 \%$ \\
\hline
\end{tabular}

\section{Comentarios de expertos}

Además de la clasificación, los expertos podían expresar sus opiniones acerca de los indicadores durante la clasificación y, al terminar, sobre el IDE en general. A continuación se presenta un resumen de las consideraciones que deben ser tenidas en cuenta durante la socialización del índice, el cálculo de los indicadores y su interpretación.

Los expertos destacan la dificultad de clasificar algunos de los indicadores en una sola dimensión, especialmente cuando esto depende del contexto. Sobre la eficiencia, uno de los expertos señala que:
Porcentaje de la matrícula en jornada única en el sector oficial

\begin{tabular}{|c|c|c|c|}
\hline \multicolumn{1}{|c|}{ D } & \multicolumn{1}{c}{ Acc } & \multicolumn{1}{c}{ Ad } & Ace \\
\hline $75 \%$ & $7 \%$ & $14 \%$ & $4 \%$ \\
\hline $76 \%$ & $6 \%$ & $18 \%$ & $0 \%$ \\
\hline $54 \%$ & $15 \%$ & $23 \%$ & $8 \%$ \\
\hline $55 \%$ & $9 \%$ & $9 \%$ & $27 \%$ \\
\hline
\end{tabular}

Tasa de reprobación en el sector oficial

\begin{tabular}{|c|c|c|c|}
\hline \multicolumn{1}{c|}{ D } & \multicolumn{1}{c}{ Acc } & \multicolumn{1}{c}{ Ad } & Ace \\
\hline $0 \%$ & $0 \%$ & $100 \%$ & $0 \%$ \\
\hline $0 \%$ & $0 \%$ & $86 \%$ & $14 \%$ \\
\hline $3 \%$ & $0 \%$ & $68 \%$ & $29 \%$ \\
\hline $3 \%$ & $0 \%$ & $52 \%$ & $45 \%$ \\
\hline
\end{tabular}

Tasa de terminación de educación primaria y secundaria por una cohorte

\begin{tabular}{|c|c|c|c|}
\hline D & \multicolumn{1}{|c|}{ Acc } & Ad & Ace \\
\hline $33 \%$ & $17 \%$ & $17 \%$ & $33 \%$ \\
\hline $0 \%$ & $0 \%$ & $43 \%$ & $57 \%$ \\
\hline $4 \%$ & $4 \%$ & $8 \%$ & $83 \%$ \\
\hline $0 \%$ & $0 \%$ & $33 \%$ & $67 \%$ \\
\hline
\end{tabular}

Indice de Paridad de Género en
Cobertura
\begin{tabular}{|c|c|c|c|}
\hline D & Acc & Ad & Ace \\
\hline $20 \%$ & $80 \%$ & $0 \%$ & $0 \%$ \\
\hline $2 \%$ & $86 \%$ & $9 \%$ & $2 \%$ \\
\hline $15 \%$ & $62 \%$ & $23 \%$ & $0 \%$ \\
\hline $0 \%$ & $75 \%$ & $0 \%$ & $25 \%$ \\
\hline
\end{tabular}

Tránsito inmediato entre niveles de educación

\begin{tabular}{|c|c|c|c|}
\hline \multicolumn{1}{c|}{ D } & \multicolumn{1}{c}{ Acc } & \multicolumn{1}{c}{ Ad } & \multicolumn{1}{c}{ Ace } \\
\hline $0 \%$ & $50 \%$ & $30 \%$ & $20 \%$ \\
\hline $13 \%$ & $27 \%$ & $40 \%$ & $20 \%$ \\
\hline $9 \%$ & $23 \%$ & $41 \%$ & $27 \%$ \\
\hline $0 \%$ & $13 \%$ & $61 \%$ & $26 \%$ \\
\hline
\end{tabular}

\begin{tabular}{|c|c|c|c|}
\hline \multicolumn{1}{c}{ D } & \multicolumn{1}{c}{ Acc } & \multicolumn{1}{c}{ Ad } & \multicolumn{1}{c}{ Ace } \\
\hline $52 \%$ & $4 \%$ & $4 \%$ & $39 \%$ \\
\hline $20 \%$ & $0 \%$ & $0 \%$ & $80 \%$ \\
\hline $20 \%$ & $0 \%$ & $20 \%$ & $60 \%$ \\
\hline $9 \%$ & $0 \%$ & $3 \%$ & $89 \%$ \\
\hline
\end{tabular}

Sería importante definir de forma más precisa la categoría Aceptabilidad, pues parece insinuar que la calidad se circunscribe a la eficiencia, entendida como el tiempo normal en que un estudiante debe tardar en completar sus estudios, sin tener en cuenta otros factores que inciden en su desarrollo normal. Vale la pena fortalecer la discusión sobre qué es y cómo medir la calidad educativa.

Al tiempo, se presentó una inquietud general frente a la pertinencia del Índice en contextos no convencionales, como la Escuela Nueva o algunas zonas rurales. El siguiente comentario plantea este asunto con gran claridad: 
También recomiendo revisar, críticamente, si el planteamiento de este índice y sus distintos indicadores tiene en cuenta una perspectiva diferencial, reconociendo que vivimos en un país pluriétnico y multicultural. ¿Cómo se sitúa el índice en las comunidades indígenas, si se tienen los mismos entendimientos para los centros etnoeducativos?, y ¿quiénes buscan preservar el derecho a una educación propia?, por ejemplo.

Los expertos también recomendaron incluir indicadores que permitan ver "condiciones de vulnerabilidad, no solo edad, sino también la permanencia de población con discapacidad, víctimas de conflicto, entre otras". Frente a este comentario es importante indicar que se ha considerado la posibilidad de incluir estas variables en el IDE, sin embargo, la información administrativa al respecto es de muy mala calidad, lo que impide que sea usada por ahora. De la misma forma, hubo un comentario que invitaba a ampliar el número de "indicadores que den cuenta de una educación más inclusiva [yendo] más allá del tema de discriminación, en un marco de derecho: el de aprender a convivir sobre la base del reconocimiento y valoración del otro". Sin embargo, no se precisa un indicador concreto ni la fuente de información que podría ser usada para su construcción.

Una crítica expresada frente a la construcción del IDE giró alrededor de que se trata de una propuesta donde se evalúan "los resultados en términos cuantitativos y no el quehacer pedagógico", lo cual puede limitar su estudio, pues "no están posibilitando alternativas reflexivas que inviten a la transformación de prácticas educativas, sino al cumplimiento de parámetros, lineamientos y el alcance de los desempeños". Sin embargo, esto depende principalmente del uso que se puede dar al IDE, por ejemplo, en otro comentario se sugiere "tener un encuentro regional o nacional para analizar los indicadores".

Durante la sección propia de cada indicador se presentaron algunos comentarios generales, como la oposición a la presentación de resultados en términos cuantitativos, que impide la reflexión sobre el quehacer pedagógico; pero también se destacaron dos tipos de opiniones más concretas: de recomendación y de incertidumbre. Las primeras se centran en tener en cuenta el contexto; en cuanto a vulnerabilidad, discapacidad, víctimas de conflicto, zona rural/urbana, edad, género; las segundas giran alrededor de la imposibilidad de clasificar algunos indicadores en una sola dimensión, en el desconocimiento de una definición precisa para cada dimensión, en el aporte que puede tener cada uno o en la forma en que se calcula.

\section{Conclusiones}

Mediante el uso de la metodología Delphi se llevó a cabo un proceso de validación de los indicadores propuestos para el Índice del Derecho a la Educación. En este proceso, los expertos llegaron a un consenso en la clasificación de los indicadores del IDE en las dimensiones, de acuerdo con el marco de las 4A propuesto por la ONU. El proceso de validación tiene una alta complejidad debido a las múltiples ópticas de los expertos; sin embargo, también resulta ser una fortaleza, en la medida en que se contempla una diversidad de opiniones.

En cuanto a las limitaciones encontradas en el proceso de validación, los expertos consultados expresan su preocupación por la pertinencia de los indicadores en algunos contextos y el enfoque cuantitativo del Índice. Sin embargo, se resalta que los indicadores son una herramienta adicional que permite disponer de información complementaria durante la toma de decisiones, con la cual es posible hacer un diagnóstico del cumplimiento del Derecho.

La validación del IDE permite tener un indicador, acordado en consenso, que funciona como referente en cuanto al nivel de cumplimiento del derecho a la educación. Estar de acuerdo en este indicador permite conocer la situación del entorno para, a partir de ésta, identificar aquellos temas que son más apremiantes. Además, permite identificar nuevas necesidades de información y así generar procesos para recolectar nuevos datos que puedan dar cuenta de las necesidades particulares.

Un indicador validado y reconocido por expertos genera un alto índice de tranquilidad a la hora de emplearlo y permite a los actores apropiarse de él. Con esto, la comunidad puede establecer metas y desarrollar un debate público que le oriente hacia el cumplimiento de este derecho. Así, se pretende que el IDE se consolide como herramienta para realizar el seguimiento al cumplimiento del Derecho a la Educación en Colombia y que sea un insumo para quienes toman las decisiones.

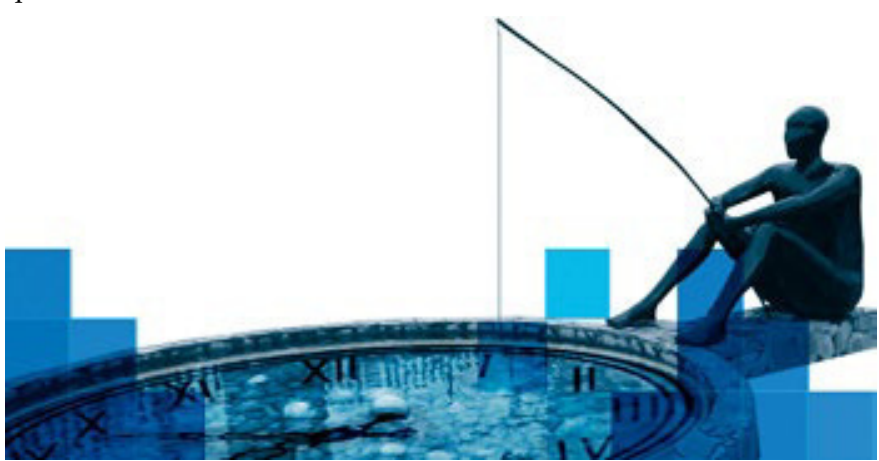




\section{Referencias}

Charro, E., Charro, E., y Niño, A. G. (2013). Enseñanza-aprendizaje por indagación de los contenidos relativos a la salud en el grado de educación primaria. Enseñanza de las ciencias: Revista de investigación y experiencias didácticas, (Extra). DOI 3812-3816.

Defensoría del Pueblo. (2004). Sistema de Seguimiento y Evaluación de la Política Pública Educativa a la Luz del Derecho a la Educación. Bogotá: Defensoría del Pueblo.

Elmendorf, D. C., y Song, L. (2015). Developing indicators for a classroom observation tool on pedagogy and technology integration: A delphi study. Computers in the Schools, 32(1), pp. 1-19.

Fundación IDEA (s.f.). Índice compuesto de eficacia de los sistemas escolares. Obtenido desde http:// fundacionidea.org/educacion/indice-compuesto-eficacia-escolar

García, V., Aquino, S., Guzmán, A., y Medina, A. (2012). El uso del método Delphi como estrategia para la valoración de indicadores de calidad en programas educativos a distancia. Revista Calidad en la Educación Superior, 3(1), pp. 200-222.

Hsu, C. C., y Sandford, B. A. (2007). The Delphi technique: making sense of consensus. Practical assessment, research \& evaluation, 12(10), pp. 1-8.

López, B., y Bañuls, V. (2017). A Delphi-based approach for detecting key e-learning trends in postgraduate education: The Spanish case. Education+ Training, 59(6).

Muñiz, L., Alonso, P., Rodríguez, L. J., y Valcke, M. (2017). Developing and validating a competence framework for secondary mathematics student teachers through a Delphi method. Journal of Education for Teaching, pp. 1-17.

ONU. (1999). El derecho a la Educación. Obtenido desde https://www.cetim.ch/legacy/es/documents/ bro11-educ-es.pdf

Pozo, M., Gutiérrez, J., y Rodríguez, C. (2007). El uso del método Delphi en la definición de los criterios para una formación de calidad en animación sociocultural y tiempo libre. Revista de investigación educativa, 5(2), pp. 351-366.

Secretaría de Educación del Distrito. (2009). Informe de Rendición de Cuentas. Bogotá Positiva. Bogotá: SED.

Slocum, N. (2003). Participatory methods toolkit. A practicioner's manual. Bruselas: King Baudouin Foundation.

Tomasevski, K. (2004). Manual on rights-based education. Global Human Rights Requirement made Simple. Bangkok: UNESCO.

UNESCO. (1990). Declaración mundial de educación para todos. Jomtien: UNESCO. . (2008). La educación inclusiva: El camino hacia el futuro. Ginebra: UNESCO.

. (2011). Informe de seguimiento de la Educación para Todos en el Mundo 2011. Una crisis encubierta: conflictos armados y educación. UNESCO.

UNICEF. (2005). Índice de los derechos de la niñez mexicana. Vigía de los derechos de la niñez mexicana, 1(1). México: UNICEF. 